\title{
Evaluation of a simple rate of spread index applied to Canadian fuel types
}

\author{
J.J. Sharples ${ }^{\mathrm{a}}$ \\ ${ }^{a}$ School of Science, UNSW Canberra, Australia \\ Email: j.sharples@adfa.edu.au
}

\begin{abstract}
The Canadian Fire Behaviour Prediction System (CFBPS) is used to predict fire behaviour across Canada and has been adapted for use in other parts of the world such as Europe and New Zealand. The CFBPS categorises fuels into fourteen different fuel types over five broad classes: coniferous, deciduous, mixedwood, slash and grass.

Rate of spread in each of the fuel types in the CFBPS is determined via calculation of the Initial Spread Index $(I S I)$, which is defined as the product of two separate empirical functions of wind speed $U$ and fuel moisture
\end{abstract} content $m$ :

$$
I S I=f(U) g(m)
$$

where

$$
f(U)=0.208 e^{0.05039 U} \quad \text { and } \quad g(m)=\left(91.9 e^{-0.1386 m}\right)\left(1+\frac{m^{5.31}}{4.93 \times 10^{7}}\right) .
$$

While wind speed is measured directly, fuel moisture content in the CFBPS is calculated using a sophisticated empirical modelling approach that accounts for both absorption and desorption processes, which are in turn modelled as functions of prior fuel moisture content, wind speed, relative humidity and air temperature. Given the overall complexity of the CFBPS rate of spread models, the question of model parsimony arises, and it is of interest to consider the possibility of more parsimonious approaches to modelling rate of spread in Canadian fuel types.

Indeed, recent research into the structure and parsimony of empirical fire spread models used in Australia has shown that for a number of different fuels types, the output of operational rate of spread models can be accurately emulated using a very simple approach. In particular, it has been shown that a single functional model, defined by two independent parameters, can accurately reproduce operational rate of spread predictions in fuels such as grass, shrubland, dry eucalypt forest, buttongrass and semi-arid mallee-heath.

It is therefore natural to wonder whether the simple approach to modelling rate of spread in Australian fuels might be more widely applicable to, for example, the fuel types in the CFBPS. In this paper we address this question by evaluating the performance of the simple modelling approach to Canadian grass fuels and to Ponderosa pine/Douglas-fir. These preliminary analyses demonstrate that the following simplified models are able to emulate predictions of the CFBPS for grass fuels (fuel type O-1) and Ponderosa pine/Douglas-fir (fuel type C-7) to within a reasonable degree of accuracy:

$$
S_{O 1}^{*}=20.74\left(\frac{\max (1, U)}{F M I+1.84}\right)^{0.74}, \quad S_{C 7}^{*}=3.064\left(\frac{\max (1, U)}{F M I+5.39}\right)^{1.98}
$$

An alternate quadratic model for the C-7 fuel type is also discussed, and some general discussion of how the simple modelling approach can be used to provide guidance on fire behaviour in more general fuel types is provided.

Keywords: Rate of spread, grassland, Ponderosa pine, Douglas-fir, model parsimony, wind, fuel moisture content 


\section{INTRODUCTION}

Estimating the forward rate of fire spread in a particular fuel type is one of the main goals of wildfire research. Pursuit of this goal dates back to approximately 100 years ago, with early efforts in the USA and Canada. In Canada, in particular, well-known researchers such as Beall, Van Wagner and Alexander invested significant time and effort into development of the Canadian Fire Behaviour Prediction System (CFBPS). The CFBPS allows for the prediction of rate of spread and fire line intensity of fires burning in a variety of fuel types ranging from grass to conifer forests. The CFBPS has also been adapted for use in other countries, such as New Zealand.

Of course, other countries such as Australia have developed models for predicting fire behaviour in their own endemic fuel types. Indeed, such models now form the basis for the new Australian Fire Danger Rating System (AFDRS), which is being developed for operational use across the Australian continent. Specifically, the AFDRS draws upon rate of spread models for the following fuel types (Cruz et al., 2015): grasslands/savannah; dry eucalypt forest; temperate shrubland; Tasmanian buttongrass; semi-arid mallee-heath;spinifex; and pine plantation.

Recent research has shown that predictions arising from rate of spread models for Australian fuel types can be accurately replicated using a very simple and almost universal fire spread model (e.g. Sharples and McRae (2013)). In particular, it has been shown that a simple two-parameter model can accurately reproduce the predictions of the more complicated operational models for grasslands, dry eucalypt forest, temperate shrubland, buttongrass and semi-arid mallee-heath (Sharples and Bahri, 2019). Moreover, Burrows et al. (2018) show that a similar simple model can be used to predict the rate of spread of fires in spinifex.

In fact, of all the fuel types in the AFDRS, pine plantation is the only one for which a simple rate of spread model hasn't been shown to apply. It is worth noting, in this respect, that the model for rate of fire spread in pine plantations in Australia (Cruz et al. 2008) draws upon North American modelling efforts (Rothermel 1972; Van Wagner, 1977; Cruz et al., 2005). The existence of a simple, yet effective, unified approach to fire spread modelling in Australian fuels prompts the question of whether a similar simplified approach might also be effective in other fuel types, such as pine plantations and those encountered in North America.

Cruz and Alexander (2019) presented an analysis of a relatively large number of wildfire observations documented in conifer forests, dry eucalypt forests and temperate shrublands, which revealed that the forward rate of fire spread is roughly $10 \%$ of the average $10-\mathrm{m}$ open wind speed. This simple rule of thumb was found to give the most accurate results for dry fuels and high wind speed conditions. Indeed, under such conditions, the error statistics were comparable to those obtained by established rate of spread models (Cruz and Alexander. 2019). However, in addition to the sensitivity to fuel moisture content and wind speed, Cruz and Alexander (2019) also determined that their simple rule of thumb was not applicable to grasslands. These findings suggest that, in general, a simple fire spread model should also possess a component that properly accounts for the influence of fuel moisture content on rate of spread, and that extra degrees of freedom should be accommodated for such a model to apply across a broader range of fuel types.

Therefore, in the present paper, we consider the applicability of a simple two-parameter model for rate of spread to Canadian fuel types. In this preliminary work, we concentrate on application of the model to only two of the fourteen fuel types that constitute the CFBPS. Specifically, we consider the grass and Ponderosa pine/Douglas-fir fuel types. We begin by outlining the structure of the rate of spread models for these two fuel types in the CFBPS before discussing the simple rate of spread model. An inter-model comparison using real meteorological data is performed to assess the applicability of the simple model to the two Canadian fuel types.

\section{RATE OF FIRE SPREAD MODELS IN THE CFBPS}

The CFBPS groups fuels into five broad classes: coniferous, deciduous, mixedwood, slash and grass. The forward rate of spread of fire burning in the various fuel types is determined via the Initial Spread Index (ISI), with each fuel type corresponding to a separate function of $I S I$ (Alexander et al. 1984). The ISI itself is a function of wind speed and fuel moisture content, the latter of which can be modelled using temperature, relative humidity and wind speed as input variables. It is worth noting that the calculation of fuel moisture content in the CFBPS accounts for both absorption and desorption processes.

Specifically, fuel moisture content $m(\%)$ is determined daily, based on the previous day's fuel moisture content 
$m_{0}$, as follows:

$$
m= \begin{cases}E_{d}+\left(m_{0}-E_{d}\right) \times 10^{-k_{d},}, & \text { if } m_{0}>E_{d} \\ E_{w}-\left(E_{w}-m_{0}\right) \times 10^{-k_{w}}, & \text { if } m_{0}<E_{w} \\ m_{0}, & \text { otherwise }\end{cases}
$$

Here $E_{d}$ is the equilibrium moisture content obtained by drying from above, and $E_{w}$ is the equilibrium moisture content obtained by wetting from below, in percent moisture content based on dry weight (Van Wagner 1987). Explicitly, $E_{d}$ and $E_{w}$ are given in terms of relative humidity $H$ and temperature $T$ as:

$$
E_{d}=0.942 H^{0.679}+11 e^{(H-100) / 10}+0.81(21.1-T)\left(1-e^{-0.115 H}\right),
$$

and

$$
E_{w}=0.618 H^{0.753}+11 e^{(H-100) / 10}+0.81(21.1-T)\left(1-e^{-0.115 H}\right) .
$$

The quantities $k_{d}$ and $k_{w}$ in equation (1) are the drying and wetting rates, respectively, and are defined as:

$$
k_{d}=0.581 k_{d}^{0} e^{0.0365 T},
$$

and

$$
k_{w}=0.581 k_{w}^{0} e^{0.0365 T}
$$

where

$$
k_{d}^{0}=0.424\left[1-\left(\frac{H}{100}\right)^{1.7}\right]+0.069 U^{0.5}\left[1-\left(\frac{H}{100}\right)^{8}\right],
$$

and

$$
k_{w}^{0}=0.424\left[1-\left(\frac{(100-H)}{100}\right)^{1.7}\right]+0.069 U^{0.5}\left[1-\left(\frac{(100-H)}{100}\right)^{8}\right]
$$

where $U$ is the wind speed. Once the fuel moisture content has been determined, the $I S I$ is calculated as the following function of wind speed and fuel moisture content:

$$
I S I=0.208 e^{0.05039 U}\left(91.9 e^{-0.1386 m}\right)\left(1+\frac{m^{5.31}}{4.93 \times 10^{7}}\right) .
$$

In this preliminary analysis, we only consider grass (fuel type O-1) and Ponderosa Pine/ Douglas-fir (fuel type C-7). The rate of fire spread in these two fuel types is given in terms of the $I S I$ as:

$$
R_{O-1}=4.88 I S I^{0.626}
$$

and

$$
R_{C-7}= \begin{cases}0.0201 I S I^{1.879}, & \text { if } I S I \leq 35 \\ 40\left(1-e^{-0.0341(I S I-20)}\right), & \text { if } I S I>35\end{cases}
$$

It is worth noting the complexity of the models for rate of spread just presented. Overall, there are around twenty model parameters that are required to determine the rate of spread in the $\mathrm{O}-1$ and $\mathrm{C}-7$ fuel types. The rate of spread models for other fuel types in the CFBPS are of a similar level of complexity. 


\section{A SIMPLE MODEL FOR RATE OF SPREAD}

Previous work (e.g. Sharples and McRae (2010)) considered the utility of the following simple dimensionless index in describing fuel moisture content. The fuel moisture index is defined as:

$$
F M I=10-0.25(T-H)
$$

where $T$ is air temperature $\left({ }^{\circ} \mathrm{C}\right)$ and $H$ is relative humidity $(\%)$. The $F M I$ has been combined with wind speed in simple functional forms, which have been shown to provide estimates of fire danger and rates of spread that are comparable to those derived from accepted models (e.g. Sharples et al. (2009); Sharples and McRae (2013); Sharples and Bahri (2019)). In this work, we extend this idea, and examine how predictions from a simple, two-parameter model for rate of fire spread, based on wind speed $U$ and $F M I$, compares to the Canadian models introduced in the previous section. The particular simple model, which we refer to as the spread index, is:

$$
S(\mu, p)=\left(\frac{\max (1, U)}{F M I+\mu}\right)^{p},
$$

where $\mu$ and $p$ are the two parameters defining the model.

The simple rate of spread index $S(\mu, p)$ can be converted into a model for rate of spread via multiplication by a dimensional scale factor, which aligns index values with actual rate of spread values (expressed in appropriate units). We will refer to the rate of spread model so obtained as

$$
S^{*}(\mu, p)=\alpha S(\mu, p),
$$

where $\alpha$ is a scale factor with units of $\mathrm{m} / \mathrm{min}$ for rate of spread in Canadian fuel types.

In the remainder of the paper, we examine how predictions of rate of spread derived from $S^{*}(\mu, p)$ compare to those derived using the CFBPS rate of spread models for grass (fuel type O-1) and Ponderosa Pine/Douglas-fir (fuel type C-7). The scale factor $\alpha$ is chosen so that the average of $S^{*}(\mu, p)$ matches that of the CFBPS models.

To facilitate the comparison between the CFBPS models and the simple model, we use half-hourly fire weather data recorded at Canberra Airport between November 2006 March 2007; that is, approximately over the course of a fire season. Note that this is not strictly in keeping with the way the CFBPS is supposed to be implemented (it is normally implemented using daily fire weather data), but is sufficient to make a comparison of the rate of spread values the system produces.

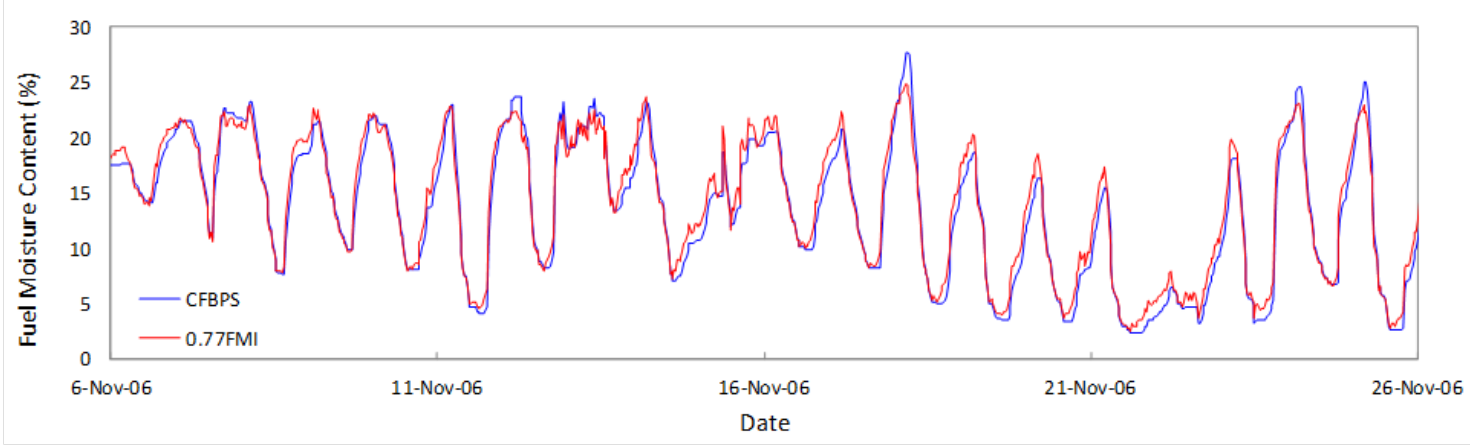

Figure 1. Time series of fuel moisture content for the 20 day period 6-26 November, 2006. The blue time series is the fuel moisture content calculated according to the CFBPS using equation (1), while the red time series is the FMI divided by 1.3 (or $0.77 F M I$ ).

\section{Results}

The first thing worth noting is how the fuel moisture index compares with fuel moisture content values calculated using equation (1). Figure 1 shows that $F M I$ (divided by 1.3) provides remarkably similar values to the fuel moisture content derived from the CFBPS. The agreement is particularly good for fuel moisture 
content values below about $20 \%$, which roughly equates to the extinction fuel moisture content; that is, the fuel moisture content above which fuels will not readily burn.

The average difference between $m$ and $0.77 F M I$ is less that $1 \%$, with a maximum difference of about $8 \%$, which occurs when $m>30 \%$. Only considering fuel moisture content values satisfying $m \leq 20 \%$, the maximum difference between $m$ and $0.77 F M I$ was only $2.8 \%$, and $0.9 \%$ on average.

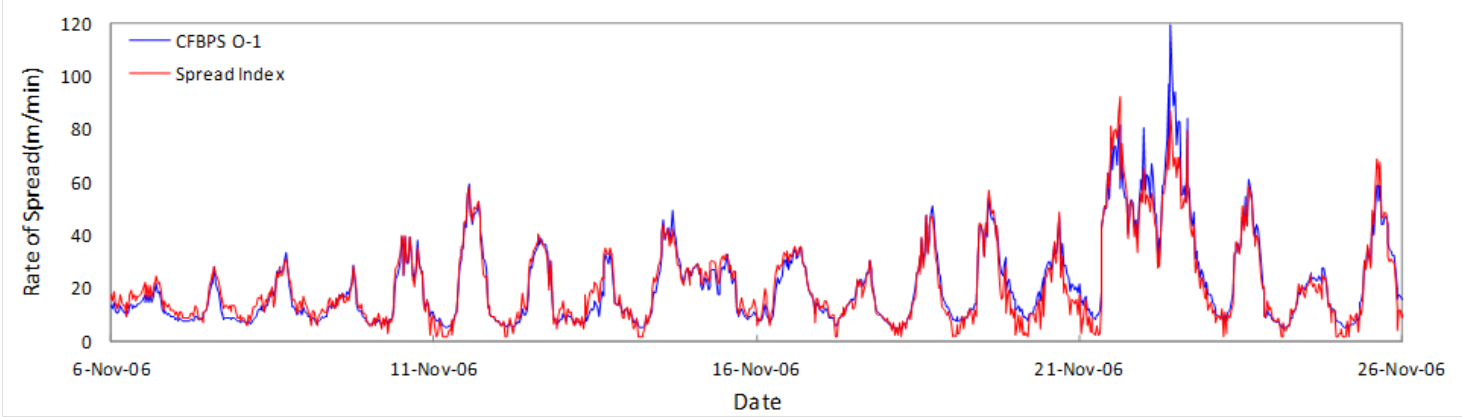

Figure 2. Time series of rate of spread $(\mathrm{m} / \mathrm{min})$ in grass (fuel type O-1) for the 20 day period 6-26 November, 2006. The red time series is rate of spread derived from equations (12) and (13) with $\alpha=20.74 \mathrm{~m} / \mathrm{min}$, $\mu=1.84$ and $p=0.74$. The blue time series is the CFBPS rate of spread $R_{O-1}$ derived using equation (9).

The parameters $\mu$ and $p$ for the simple model given by equations (12) and (13), were fitted to the CFBPS O-1 fuel type rate of spread model using least squares regression (minimising the RMS difference). This yielded the simple model:

$$
S_{O 1}^{*}=20.74\left(\frac{\max (1, U)}{F M I+1.84}\right)^{0.74},
$$

The model (14) was used to derive the red rate of spread time series in Figure 2, which as can be seen, compares favourably to the rate of spread time series derived using the CFBPS model (equation (9)). On average, the difference between the rate of spread predictions using the simple index and those obtained using the CFBPS model is only $2.4 \mathrm{~m} / \mathrm{min}$, while the maximum difference is $32.5 \mathrm{~m} / \mathrm{min}$. The maximum difference coincides with hot, dry and extremely windy conditions, which it should be noted coincides with conditions that likely fall outside the calibration regime for the CFBPS.
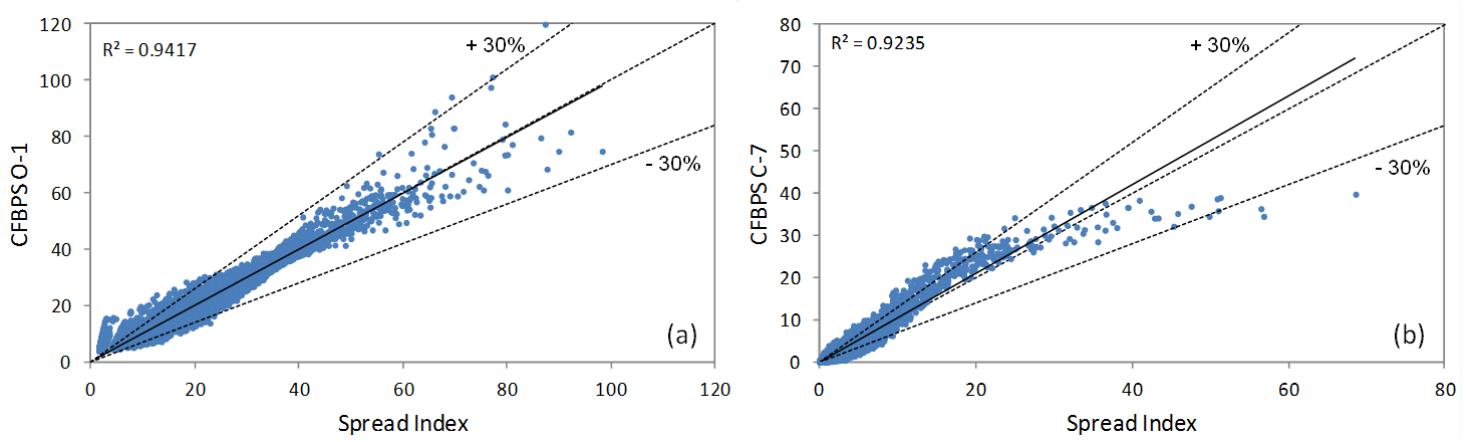

Figure 3. Scatter plots of (a) CFBPS O-1 model predictions plotted against $S_{O 1}^{*}$ (equation (14)); and (b) CFBPS C-7 model predictions plotted against $S_{C 7}^{*}$ (equation (15)). The dashed lines are the lines of perfect agreement and the $\pm 30 \%$ error lines, while the solid black line is the linear regression line for the respective data sets (blue points), with associated $R^{2}$ values provided in the top left corners of the panels.

Figure 3a shows the rate of spread in grass predicted by the CFBPS plotted against rate of spread values obtained using the simple model. The plot demonstrates that the simple model produces rate of spread values 
that are mostly within $\pm 30 \%$ of the values given by the CFBPS, which is less than the $\pm 35 \%$ benchmark set by Cruz and Alexander (2013). Moreover, the plot shows that the simple model is unbiased compared to the CFBPS model.

Figure 3 b shows the analogous plot for fire spread in Ponderosa pine/Douglas-fir (C-7 fuel type). The simple model determined through least squares regression in this case was:

$$
S_{C 7}^{*}=3.064\left(\frac{\max (1, U)}{F M I+5.39}\right)^{1.98},
$$

Unlike the O-1 fuel type, there is an overall bias of the simple model compared with the CFBPS model. Moreover, the CFBPS model plateaus in comparison to the simple model for rates of spread above about $25 \mathrm{~m} / \mathrm{min}$; hence there is a nonlinear relationship between the CFBPS C-7 rate of spread model and the corresponding simple model.

Based on this observation, the simple spread model was transformed using a quadratic function. Least squares regression analysis was again used to determine an optimal fit with the predictions of the CFBPS C-7 model. The model found through such analysis was the following:

$$
S_{C 7}^{Q}=-0.07723\left(S_{C 7}^{*}\right)^{2}+4.31341 S_{C 7}^{*}-0.50949
$$

where $S_{C 7}^{*}$ is given by equation (15).

Figure 4 shows the rate of spread in C-7 fuels predicted by the CFBPS plotted against rate of spread values obtained using the quadratic model $S_{C 7}^{Q}$ defined by equation (16). This plot that the quadratic model is unbiased compared to the CFBPS model, and produces rate of spread values that are mostly within $\pm 30 \%$ of the values given by the CFBPS. Figure 5 illustrates how the time series of the quadratic model compares with that of the CFBPS C-7 model. The average difference between values of $S_{C 7}^{Q}$ and $R_{C-7}$ is $0.9 \mathrm{~m} / \mathrm{min}$, while the maximum difference is $9.5 \mathrm{~m} / \mathrm{min}$.

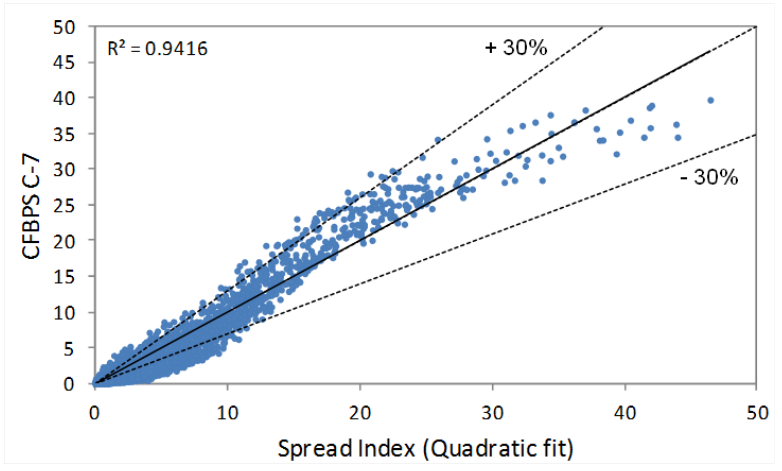

Figure 4. Scatter plot of the CFBPS C-7 model predictions plotted against the quadratic model $S_{C 7}^{Q}$ (equation (16)). The dashed lines are the lines of perfect agreement and the $\pm 30 \%$ error lines, while the solid black line is the linear regression line for the data (blue points), with associated $R^{2}$ value provided in the top left corner.

\section{Discussion And Conclusions}

A simple index has been shown to effectively emulate predictions of rate of spread in two Canadian fuel types: grass and Ponderosa pine/Douglas-fir. Indeed, the two parameter model was able to provide very similar estimates of rate of spread to models that have over twenty parameters. For the C-7 fuel type, a quadratic function of the simple spread index was able to produce even more accurate estimates of rate of spread - such a model still only has five model parameters.

Given that the same simple index has previously been shown to accurately reproduce rate of spread predictions in Australian fuel types, this study provides further evidence that the simple spread index is useful as a universal measure for fire rate of spread.

While it could be argued that the availability of modern computers makes the issue of model complexity largely irrelevant, the fact that rates of spread across a wide range of fuel types can be accurately modelled using a 


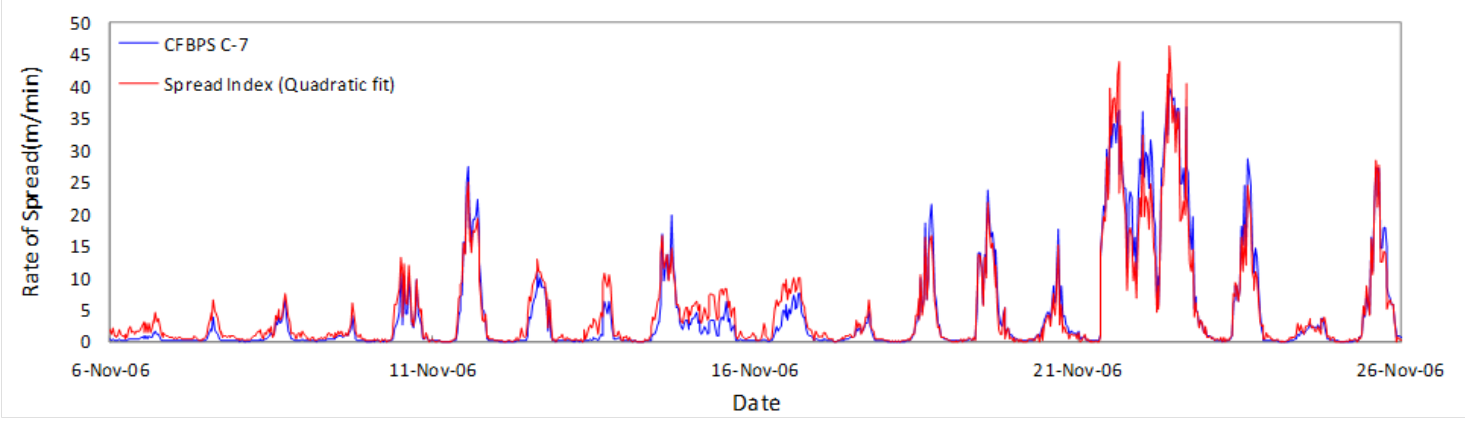

Figure 5. Time series of rate of spread (m/min) in Ponderosa pine/Douglas-fir (fuel type C-7) for the 20 day period 6-26 November, 2006. The red time series is rate of spread derived from equation (16), while the blue time series is the CFBPS rate of spread $R_{C-7}$ derived using equation (10).

simple, unified functional form provides insight into the the physical processes that are able to be resolved in empirical modelling approaches. Moreover, the simple index is more conceptually and pedagogically appealing, as it it provides transparent theoretical confirmation of the intuitive notion of fire spread that most firefighters possess; namely that strong winds and low fuel moisture content results in more rapid fire spread.

It remains to be seen how the simple index performs in the other twelve fuel types of the CFBPS and in other fuel types found elsewhere in the world. This will be pursued in further work.

\section{REFERENCES}

Alexander, M., B. Lawson, B. Stocks, and C. Van Wagner (1984). User guide to the canadian forest fire behavior prediction system: rate of spread relationships. General technical report, Canadian Forestry Service.

Burrows, N., A. Gill, and J. Sharples (2018). Development and validation of a model for predicting fire behaviour in spinifex grasslands of arid Australia. International Journal of Wildland Fire 27(4), 271-279.

Cruz, M. and M. Alexander (2013). Uncertainty associated with model predictions of surface and crown fire rates of spread. Environmental Modelling \& Software 47, 16-28.

Cruz, M. and M. Alexander (2019). The $10 \%$ wind speed rule of thumb for estimating a wildfires forward rate of spread in forests and shrublands. Annals of Forest Science 76(2), 44.

Cruz, M., M. Alexander, and P. Fernandes (2008). Development of a model system to predict wildfire behaviour in pine plantations. Australian Forestry 71(2), 113-121.

Cruz, M., M. Alexander, and R. Wakimoto (2005). Development and testing of models for predicting crown fire rate of spread in conifer forest stands. Canadian Journal of Forest Research 35(7), 1626-1639.

Cruz, M., J. Gould, M. Alexander, A. Sullivan, W. McCaw, and S. Matthews (2015). A guide to rate of fire spread models for Australian vegetation. Australasian Fire and Emergency Service Authorities Council Limited and Commonwealth Scientific and Industrial Research Organisation.

Rothermel, R. (1972). A mathematical model for predicting fire spread in wildland fuels. Research Paper INT-115, USDA Forest Service, Intermountain Forest and Range Experiment Station.

Sharples, J. and M. Bahri (2019). A universal rate of spread index for australian fuel types. International Journal of Wildland Fire (submitted).

Sharples, J. and R. McRae (2010). Evaluation of a very simple model for predicting the moisture content of eucalypt litter. International Journal of Wildland Fire 20(8), 1000-1005.

Sharples, J. and R. McRae (2013). A fire spread index for grassland fuels. In J. Piantadosi, R. Anderssen, and J. Boland (Eds.), MODSIM2013, 20th International Congress on Modelling and Simulation, pp. 249-255. Modelling and Simulation Society of Australia and New Zealand.

Sharples, J., R. McRae, R. Weber, and A. Gill (2009). A simple index for assessing fire danger rating. Environmental Modelling \& Software 24(6), 764-774.

Van Wagner, C. (1977). Conditions for the start and spread of crown fire. Canadian Journal of Forest Research 7(1), 23-34.

Van Wagner, C. (1987). Development and structure of the Canadian forest fire weather index system, Volume 35. Canadian Forestry Service, Ottawa. 